\title{
Characterisation of cardiomyopathy by cardiac and aortic magnetic resonance in patients new to hemodialysis
}

\author{
Aghogho Odudu ${ }^{1,2,3,4}$ (D) Mohamed Tarek Eldehni ${ }^{3,4} \cdot$ Gerry P. McCann $^{5,6}$. \\ Mark A. Horsfield ${ }^{5,6}$ • Tobias Breidthardt ${ }^{4,7}$ • Christopher W. McIntyre M,8 $^{3,8}$
}

Received: 6 May 2015 /Revised: 12 September 2015 / Accepted: 28 October 2015 /Published online: 17 December 2015

(C) The Author(s) 2015. This article is published with open access at Springerlink.com

\begin{abstract}
Objectives Cardiomyopathy is a key factor in accelerated cardiovascular mortality in haemodialysis (HD) patients. We aimed to phenotype cardiac and vascular dysfunction by tagged cardiovascular magnetic resonance (CMR) imaging in patients recently commencing HD.

Methods Fifty-four HD patients and 29 age and sex-matched controls without kidney disease were studied. Left ventricular (LV) mass, volumes, ejection fraction (EF), concentric remodelling, peak-systolic circumferential strain (PSS), peak diastolic strain rate (PDSR), LV dyssynchrony, aortic distensibility and aortic pulse wave velocity were determined.

Results Global systolic function was reduced (EF $51 \pm 10 \%$, HD versus $59 \pm 5 \%$, controls, $p<0.001$; PSS $15.9 \pm 3.7 \%$ versus 19.5 $\pm 3.3 \%, p<0.001$ ). Diastolic function was decreased (PDSR 1.07 $\pm 0.33 \mathrm{~s}^{-1}$ versus $\left.1.31 \pm 0.38 \mathrm{~s}^{-1}, p=0.003\right)$. LV mass index was increased $\left(63[54,79] \mathrm{g} / \mathrm{m}^{2}\right.$ versus $\left.46[42,53] \mathrm{g} / \mathrm{m}^{2}, p<0.001\right)$. Anteroseptal reductions in PSS were apparent. These abnormalities remained prevalent in the subset of HD patients with
\end{abstract}

preserved $\mathrm{EF}>50 \%(\mathrm{n}=35)$ and the subset of HD patients without diabetes $(n=40)$. LV dyssynchrony was inversely correlated to diastolic function, EF and aortic distensibility. Diastolic function was inversely correlated to LV dyssynchrony, concentric remodelling, age and aortic pulse wave velocity.

Conclusion Patients new to HD have multiple cardiac and aortic abnormalities as characterised by tagged CMR. Cardioprotective interventions are required from initiation of therapy. Key Points

- First characterisation of cardiomyopathy by tagged CMR in haemodialysis patients.

- Diastolic function was correlated to LV dyssynchrony, concentric remodelling and aortic $P W V$.

- Reductions in strain localised to the septal and anterior wall.

- Bioimpedance measures were unrelated to LV strain, suggesting volume-independent pathogenetic mechanisms.

- Multiple abnormalities persisted in the HD patient subset with preserved EF or without diabetes.
Electronic supplementary material The online version of this article (doi:10.1007/s00330-015-4096-2) contains supplementary material, which is available to authorized users.

Aghogho Odudu a.odudu@manchester.ac.uk

1 Institute of Cardiovascular Sciences, University of Manchester, Manchester, UK

2 National Institute for Health Research Clinical Lecturer in Nephrology, Institute of Cardiovascular Sciences, Manchester Academic Health Science Centre, University of Manchester, Core Technology Facility, 46 Grafton Street, Manchester M13 9NT, UK

3 Division of Medical Sciences, University of Nottingham, Nottingham, UK
4 Department of Renal Medicine, Royal Derby Hospital, Derby, UK

5 Department of Cardiovascular Sciences, University of Leicester, Leicester, UK

6 National Institute for Health Research Leicester Cardiovascular Biomedical Research Unit, Glenfield Hospital, Leicester, UK

7 Clinic for Transplant-Immunology and Nephrology, University Hospital Basel, Basel, Switzerland

8 Division of Nephrology, Schulich School of Medicine and Dentistry, University of Western Ontario, London, Canada 
Keywords Aortic distensibility · Dyssynchrony · Cardiac magnetic resonance $\cdot$ Cardiomyopathy $\cdot$ Hemodialysis

\section{Introduction}

Abnormal cardiac and vascular structure and function play a key part in excessive cardiovascular events and premature mortality experienced by patients with end-stage renal disease using haemodialysis (HD). This cannot wholly be attributed to the high burden of atherosclerotic risk factors. Factors specific to chronic kidney disease (CKD) such as anaemia, hyperphosphatemia and vascular calcification are well-recognized to cause left ventricular dilatation and hypertrophy [1]. It is also known that the HD procedure itself contributes to development of cardiomyopathy by inducing hypotension and cardiac ischemia, as detected by early transient reductions in ventricular deformation that precede global dysfunction and increased mortality. Drug-based therapies that reduce cardiovascular morbidity in the CKD population have been ineffective in those using HD [2]. The development of cardiomyopathy might be prevented by early application of HD-based interventions such as cool dialysate or an extended length of HD treatment [3, 4].

Adverse changes in left ventricular (LV) geometry and function after cardiac injury are termed remodelling. At a cellular level, remodelling is characterised by cardiomyocyte hypertrophy, interstitial fibrosis, apoptosis and capillary rarefaction. Cardiovascular magnetic resonance (CMR) imaging is the reference standard technique for analysis of LV mass, volumes and geometry. The LV mass-to-volume ratio indexes LV wall thickness to cavity size, categorizing LV geometry as concentric remodelling, concentric hypertrophy or eccentric hypertrophy. The type of geometry has been noted to prognosticate cardiovascular risk beyond that by LV hypertrophy alone in HD patients and large general population studies $[5,6]$. In addition, CMR images of the left ventricle (LV) can be magnetically 'tagged' with a grid pattern. The motion of these tags can be tracked by software to quantify the magnitude (strain) and velocity (strain rate) of tissue deformation, providing an earlier marker of LV dysfunction than the ejection fraction (EF) [7].

Prior CMR data reported HD patients with several years of exposure to HD, using gadolinium contrast media that are now contraindicated for patients with impaired kidney function. Such studies are limited by prevalence-incidence bias which is particularly relevant as mortality within the first 6 months of starting HD is markedly increased [8]. There are few studies in patients new to HD using indices that might detect abnormalities earlier, such as strain or dyssynchrony [9]. We aimed to phenotype cardiac abnormalities in an unselected incident HD population with non-contrast tagged CMR to determine the characteristics and severity of HD-associated cardiomyopathy in the early phase of a patient's time using HD.

\section{Materials and methods}

\section{Subjects}

This multi-centre study was approved by the institutional review board and conducted in accordance with the Declaration of Helsinki. All participants gave written informed consent. The HD patients were recruited from four geographically local university teaching hospitals as part of a clinical trial of an intervention to prevent cardiomyopathy, with these data acquired prior to any trial interventions. Inclusion criteria were:

1. Age $\geq 16$ years

2. Having HD treatment three times per week.

3. Willing and able to provide consent.

4. Exposure to haemodialysis for $\leq 180$ days.

Exclusion criteria were:

1. Contraindications for CMR

2. Inability to tolerate CMR due to claustrophobia

3. New York Heart Association grade IV heart failure

4. Pregnancy or lactating

5. Mental incapacity to consent

Healthy volunteers were recruited through a local clinical research volunteer program. Their inclusion criteria were:

1. Age $\geq 16$ years.

2. Willing and able to provide consent.

Exclusion criteria were:

1. Contraindications for CMR

2. Inability to tolerate CMR due to claustrophobia

3. History of kidney disease, heart disease or cardiomyopathy

4. Mental incapacity to consent

\section{HD characteristics}

Patients were treated with in-centre HD treatments three times per week, with treatment times of 3.5-4 hours using low-flux dialyzers (LOPS 18/20; Braun Medical Ltd, Sheffield, UK). Dialysate temperature was $36-37{ }^{\circ} \mathrm{C}$. Dialysate composition was sodium $136-138 \mathrm{mmol} / \mathrm{l}$; potassium $2 \mathrm{mmol} / \mathrm{l}$; calcium 
$1.25 \mathrm{mmol} / \mathrm{l}$; magnesium $0.5 \mathrm{mmol} / \mathrm{l}$; bicarbonate $32-38$ $\mathrm{mmol} / \mathrm{l}$; glucose $5.6 \mathrm{mmol} / \mathrm{l}$ and acetate $3 \mathrm{mmol} / \mathrm{l}$. Dialysate flow rate was $500 \mathrm{~mL} / \mathrm{min}$ and blood pump speed was 250 $450 \mathrm{~mL} / \mathrm{min}$ using anticoagulation by unfractionated heparin. Dry weight was prescribed by the clinical team according to local protocol to achieve an equilibrated $\mathrm{Kt} / \mathrm{V}_{\text {urea }}>1.1$ and standardized $\mathrm{Kt} / \mathrm{V}_{\text {urea }}$ of $>2.0$.

\section{Data collection}

Imaging by CMR was timed on a midweek post-dialysis day avoiding the long break in dialysis after the weekend, when patients are most hypervolemic [10]. Studies were conducted using a 1.5-Tesla scanner (GE Signa HDxt, GE Healthcare, Milwaukee, USA) with an 8-channel phased-array receiver coil, 12-20-second end-expiratory breath-holds and retrospective electrocardiographic (ECG) gating [11]. Steady-state free precession (SSFP) cine images were acquired in 2-, 3- and 4chamber views to plan a stack of $8-\mathrm{mm}$ short-axis slices with a 2-mm interslice gap, spanning the cardiac apex through the ventricular base. Imaging parameters as detailed in Table 1 were optimised with a minimal field of view to achieve a typical in-plane spatial resolution $<2 \mathrm{~mm}$ and temporal resolution $<50 \mathrm{~ms}$. Radiofrequency tagging by a spatial modulation of magnetization (SPAMM) sequence with gradient recalled-echo readout was applied at basal, mid-ventricular and apical levels, typically achieving temporal resolution $<40 \mathrm{~ms}$. Multi-plane localizer images were used to plan an oblique sagittal SSFP cine of the ascending and descending aorta. An axial SSFP cine was acquired at the level of the pulmonary artery bifurcation to allow assessment of aortic distensibility. An orthogonal high temporal resolution $(<10$ $\mathrm{ms})$ through-plane velocity-encoded sequence was acquired to allow assessment of pulse-wave velocity.

\section{Body composition}

Volume status was assessed by segmental multiple-frequency bioelectrical impedance measurements, using tetrapolar 8point tactile electrodes (InBody S20, Biospace, Seoul, South Korea) [12]. Hypervolemia was estimated by the ratio of extracellular water to total body water [13].

\section{Biochemical markers}

Serum samples for determination of high-sensitivity troponin-T and NT-proBNP were taken immediately after insertion of access needles on HD on the day preceding the CMR study. Samples were promptly centrifuged and analysed using the Elecsys 2010 system (Roche Diagnostics, Switzerland). The limits of detection of the troponin- $\mathrm{T}$ assay are $0.005 \mu \mathrm{g} / \mathrm{L}$, the 99 th percentile $0.014 \mu \mathrm{g} / \mathrm{L}$ and the $10 \%$ co-efficient of variation is 0.013 $\mu \mathrm{g} / \mathrm{L}[14]$. The limits of detection of the NT-proBNP assay is 0.6 $\mathrm{pmol} / \mathrm{l}$ and the interassay co-efficient of variation is $3.9 \%$.

Table 1 Typical CMR acquisition parameters

\begin{tabular}{|c|c|c|c|c|}
\hline Parameters & Ventricular SSFP-cines & $\begin{array}{l}\text { Ventricular SPAMM } \\
\text { tagging }\end{array}$ & $\begin{array}{l}\text { Aortic SSFP-cines } \\
\text { for distensibility }\end{array}$ & $\begin{array}{l}\text { Aortic velocity-encoded } \\
\text { for PWV }\end{array}$ \\
\hline Respiratory compensation & breath-hold & breath-hold & breath-hold & free breathing \\
\hline Acquisition time (s) & $12-18$ & $12-18$ & $12-18$ & $240-360$ \\
\hline ECG gating & retrospective & retrospective & retrospective & retrospective \\
\hline TR: repetition time (ms) & 3.2 & 6.7 & 3.2 & 6.7 \\
\hline TE: echo time (ms) & 1.7 & 3.1 & 1.7 & 4.5 \\
\hline Voxel size (mm) & $1.7 \times 1.7 \times 8$ & $1.7 \times 2.4 \times 10$ & $1.3 \times 2.5 \times 5$ & $1.3 \times 2.5 \times 5$ \\
\hline Slice thickness (mm) & 8 & 10 & 5 & 5 \\
\hline Interslice gap (mm) & 2 & $6-12$ & NA & NA \\
\hline Typical field-of-view (mm) & 380 & 380 & 320 & 320 \\
\hline Matrix & $224 \times 224$ & $224 \times 160$ & $256 \times 128$ & $256 \times 128$ \\
\hline Parallel imaging & enabled & enabled & enabled & enabled \\
\hline View-sharing & Yes & Yes & Yes & Yes \\
\hline Flip angle (degrees) & 60 & 12 & 60 & 30 \\
\hline Number of signal averages & 1 & 1 & 1 & 4 \\
\hline Lines per segment & 16 & 10 & 16 & 1 \\
\hline Reconstructed phases & 30 & 30 & 30 & 100 \\
\hline Temporal resolution (ms) & $20-50$ & $20-40$ & $20-50$ & $<10$ \\
\hline $\operatorname{VENC}(\mathrm{cm} / \mathrm{s})$ & & & & $150-200$ \\
\hline
\end{tabular}




\section{Data analysis}

All CMR analyses were performed offline in a blinded fashion. End-systolic and end-diastolic LV volumes and end-diastolic LV mass were determined using cvi42 software, version 4.0.2 (Circle Cardiovascular Imaging, Calgary, Canada). The LV contours were drawn manually excluding papillary muscles and trabeculations in accordance with previously validated methods proven to be more reproducible than alternative techniques $[6,15]$. LV mass was calculated by taking the difference between the end-diastolic endocardial and epicardial volume multiplied by $1.05 \mathrm{~g} / \mathrm{mL}$, which is the density of myocardium. LV concentric remodelling was determined by the LV mass/ volume ratio where a higher ratio corresponds to greater concentric remodelling. Values were indexed to body surface area using the method described by Mosteller [16].

\section{Strain analysis}

Tagged LV short-axis images were analysed using validated software as previously described [17]. The process is depicted in Fig. 1. Peak-systolic strain (PSS), peak-systolic strain-rate (PSSR) and peak diastolic strain-rate (PDSR) were determined. The standard deviation of segmental time-to-peak strain (SD-TTP) normalised to a heart rate of 70 beats per minute was calculated as a measure of LV dyssynchrony as previously described [18]. Data were analysed in the 16segment LV model recommended by the American Heart Association and averaged per short-axis slice and across all segments to determine global LV strains [19] (Fig. 1).

\section{Aortic distensibility}

The ascending and descending aortas were manually outlined with semi-automated propagation into all cardiac phases using the Jim software package (version 6, Xinapse Systems, UK). Aortic distensibility was calculated from the SSFP cines as (maximum aortic area - minimum aortic area) / (minimum aortic area $\mathrm{x} \Delta \mathrm{P}$ ), where $\Delta \mathrm{P}$ is the average of three brachial pulse pressure readings performed during CMR (Fig. 2) [20]. Aortic arch pulse wave velocity (PWV) was calculated as the distance around the arch between the ascending and
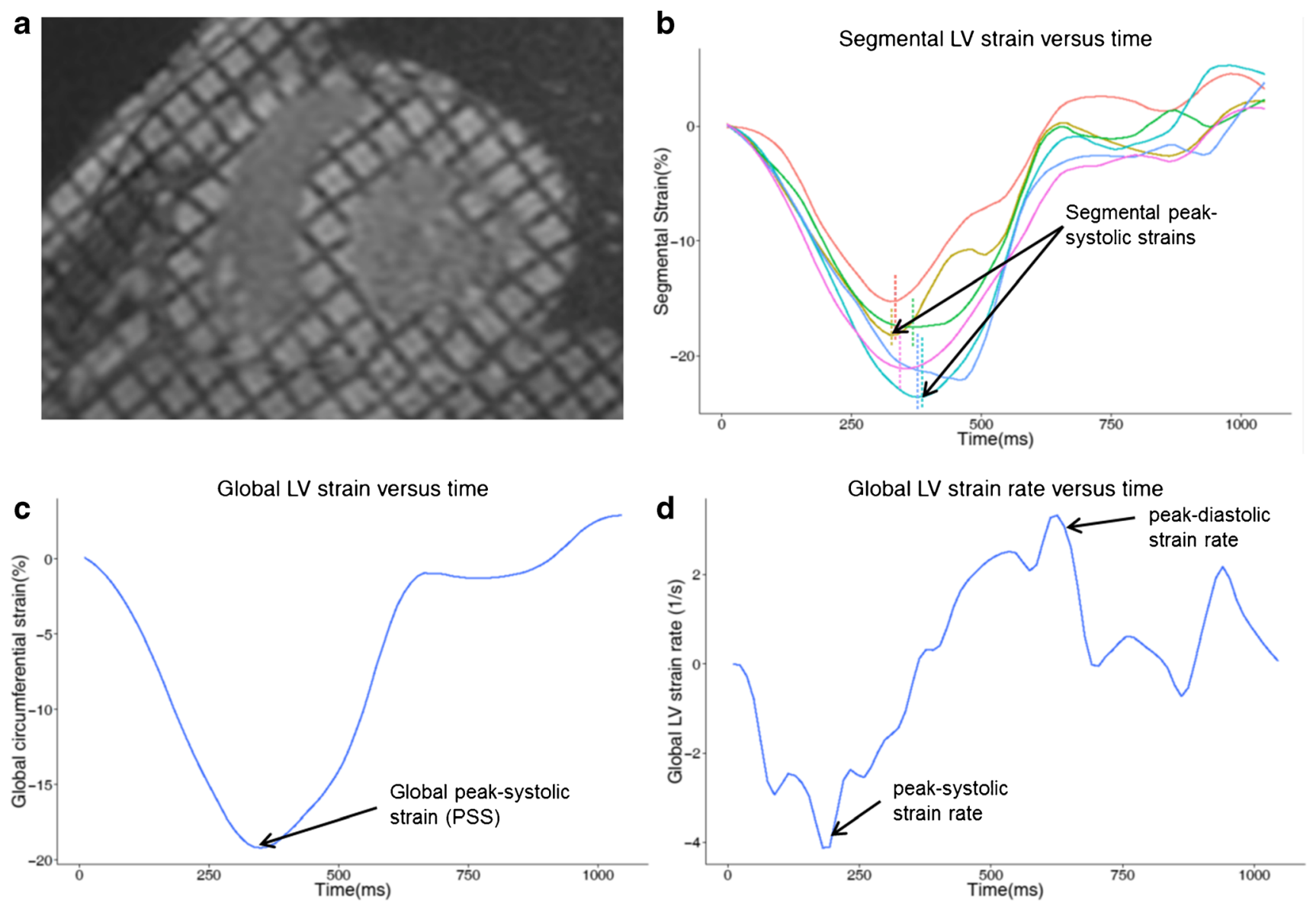

Fig. $1 \mathrm{LV}$ circumferential strain analysis in a typical study participant. Tagged LV short-axis images had manual endocardial and epicardial border tracing in a single frame, with automated propagation of the contours to all other frames to generate LV segmental strain curves $(B)$ recording

both peak strain and the time-to-peak strain (dotted lines, $B)$. Segmental strains were averaged to determine global LV strain $(C)$ and strain-rate $(D)$ curves from which peak-systolic and diastolic values were used as study outcomes 
Fig. 2 Determination of aortic distensibility in the same study participant as in Figure 2. An oblique sagittal image of the thoracic aorta $(A)$ was used to determine the pulse-wave propagation distance (solid line, $A$ ) at the level of the right pulmonary artery (dotted line, $A$ ). Orthogonal axial SSFP cines $(B)$ and velocity encoded images $(C)$ were used to contour the ascending and descending aorta to derive traces of aortic area (upper panel in $D$ ) and aortic blood flow (lower panel in $D$ ) versus time. Aortic distensibility was determined by dividing the maximal area change by pulse-pressure. Aortic arch pulse wave velocity was calculated by dividing the pulse-wave propagation distance by the transit delay between these positions determined by cross-correlation of the flow velocity profiles (lower panel in $D$ )
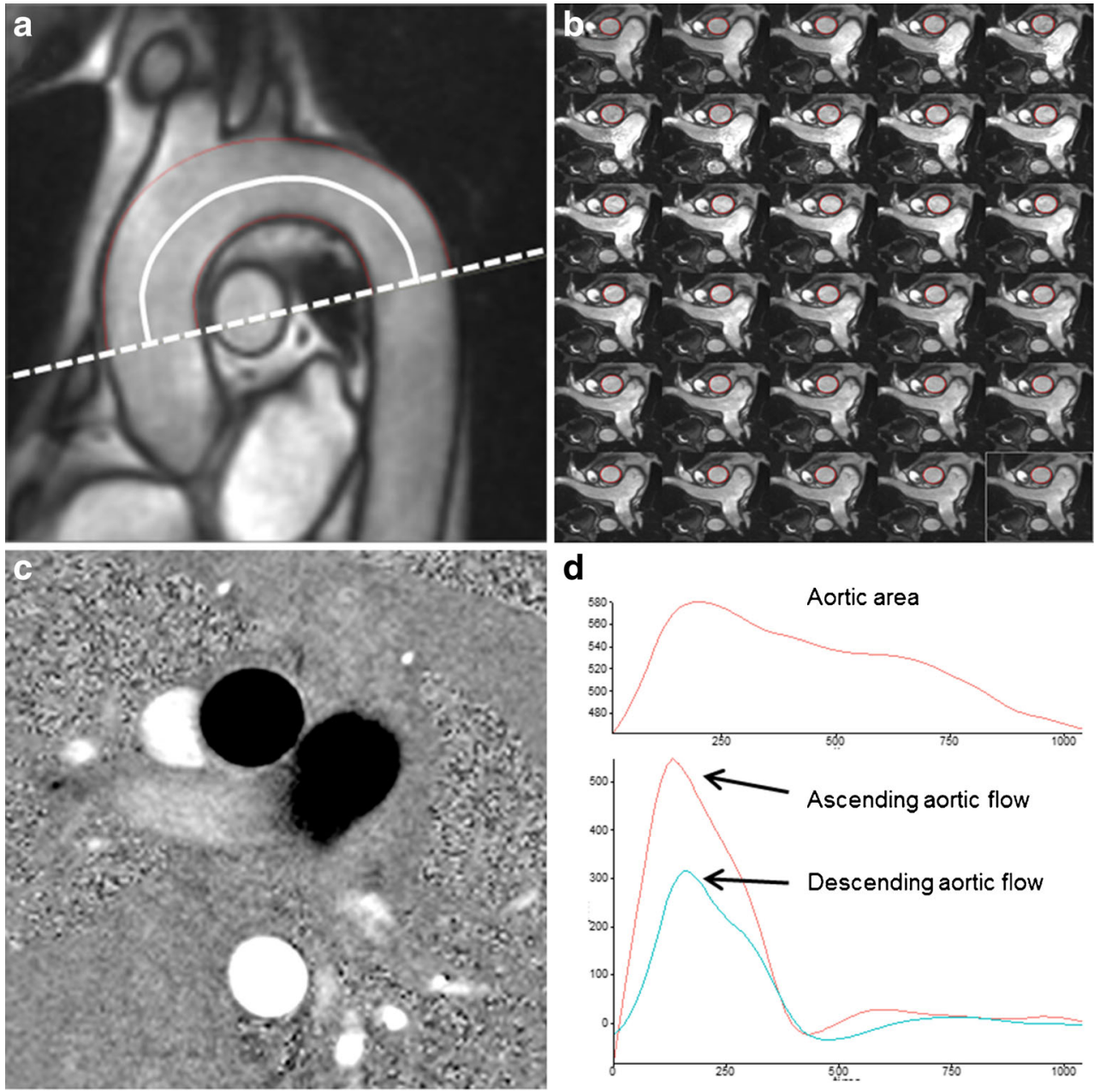

d
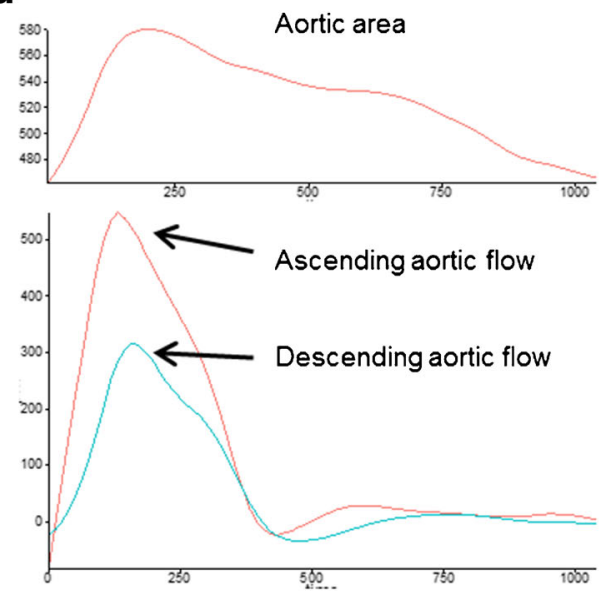

descending aorta positions in the axial slice, divided by the time delay for arrival of the pulse pressure wave between these positions (Fig. 3) [21]. The distance around the arch was assessed from an oblique sagittal view, and the time delay was calculated using cross-correlation of the flow velocity profiles generated by the velocity-encoded acquisition (Fig. 3a, d).

\section{Statistical analysis}

Continuous data were tested for normality and summarized using mean $\pm \mathrm{SD}$ or median [25th, 75 th percentile]. Differences between groups were determined using the MannWhitney or t-test as appropriate. Categorical data were expressed as counts and percentages and analysed by the Chi-squared test. Continuous variables with right-skewed distributions were logarithmically transformed prior to applying Pearson's test to assess relationships between continuous variables. The Benjamini and Hochberg procedure constrained the study-wise false discovery rate to a two-sided significance of 0.05 with adjusted $P$ values presented [22]. SPSS version 21.0 was used for all analyses.

\section{Results}

The participant flowchart is in Fig. 3. Eighty-three participants were studied; 54 patients within 6 months of commencing HD and 29 age- and sex-matched healthy volunteers. The causes of end-stage renal disease in HD patients were diabetes mellitus 17 $\%(14 / 54)$; glomerulonephritis $8 \%$ (7/54); interstitial nephritis 7 $\%(6 / 54)$; vasculitis $5 \%(4 / 54)$; polycystic kidney disease $4 \%$ (3/54); ischemic nephropathy or malignant hypertension $7 \%(6 /$ 54); light chain deposition disorder $5 \%$ (4/54) and unknown or others $12 \%(10 / 54)$. Subject characteristics and CMR findings are presented in Table 2. The groups were well matched for age, sex, body mass index, body surface area, and smoking status. Most HD patients were treated for hypertension (78 \%, 42/54) and $26 \%(14 / 54)$ were treated with a renin-angiotensinaldosterone system antagonist.

\section{Image quality}

Eight HD patients did not tolerate long breath-holds and were managed by minimising the field of view and increasing the lines per segment to allow a breath-hold less than 15 seconds. Only 1 HD patient had significant arrhythmia which was 
Fig. 3 Study participant flowchart

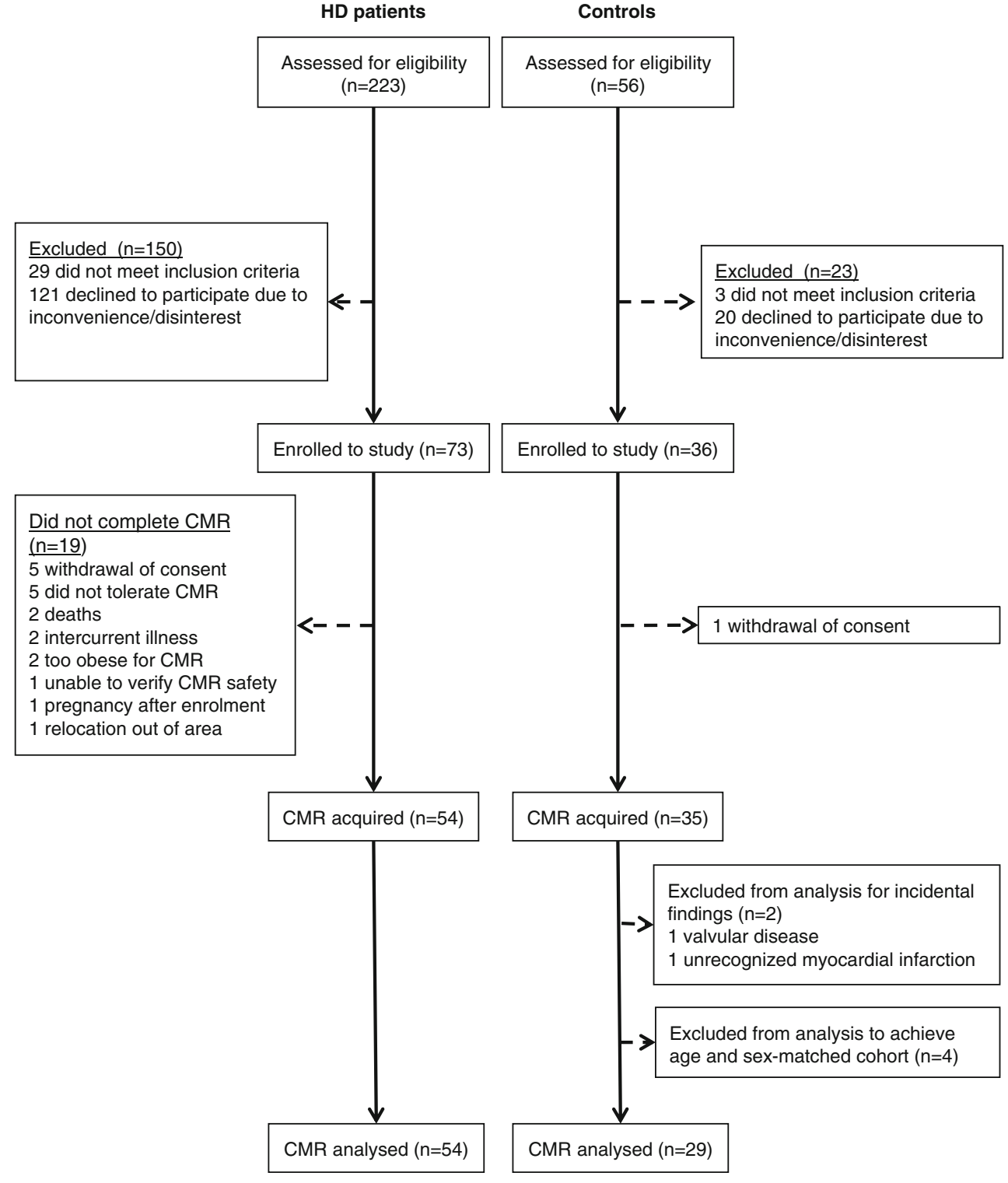

managed by increasing the arrhythmia rejection window and prospective ECG gating. All cases were of diagnostic quality as assessed by an experienced CMR operator.

\section{Global systolic and diastolic function}

Global LV systolic function was reduced in HD patients both by EF and PSS (EF $51.5 \% \pm 11.0$ in HD patients versus 58.5 $\% \pm 5.4$ in control subjects, $p<0.001$; PSS $15.9 \% \pm 3.7$ versus $19.5 \% \pm 3.3, p<0.001)$. Systolic function by global PSS was inversely correlated to LV dyssynchrony $(\mathrm{r}=-0.51, p<0.001)$, concentric remodelling $(\mathrm{r}=-0.37, p=0.005)$ and troponin- $\mathrm{T}$ $(\mathrm{r}=-0.41, p=0.03)$. Diastolic function was reduced in HD patients (PDSR $1.07 \pm 0.33 \mathrm{~s}^{-1}$ in HD patients versus $1.31 \pm 0.38$ $\mathrm{s}^{-1}$ in control subjects, $\left.p=0.003\right)$. Diastolic function was inversely correlated to LV dyssynchrony $(\mathrm{r}=-0.51, p<0.001)$, concentric remodelling, aortic PWV $(\mathrm{r}=-0.48, p=0.04)$ and age $(\mathrm{r}=-0.31, p=0.02)$.

\section{LV mass and geometry}

LV mass was increased in HD patients, $(63[54,79]$ versus 46 $\left.[42,53] \mathrm{g} / \mathrm{m}^{2}, p<0.001\right)$ with greater LV concentric remodelling (LV mass/volume ratio $0.76[0.64,0.86]$ versus 0.65 $[0.55,0.71], p=0.001$, Table 2).

\section{Regional heterogeneity of LV strain}

Systolic function by PSS was significantly reduced in basal, mid-cavity and apical short-axis slices in HD patients compared to NCs (Table 2). Significant regional reductions in PSS in HD patients were seen in 7 of $16 \mathrm{LV}$ segments, 
Table 2 Subject characteristics and CMR parameters

\begin{tabular}{|c|c|c|c|}
\hline & $\mathrm{HD}(\mathrm{n}=54)$ & Controls $(n=29)$ & $p$ value \\
\hline Age (years) & $57 \pm 15$ & $56 \pm 17$ & 0.7 \\
\hline Female $(\%)$ & $15(28)$ & $8(28)$ & 1 \\
\hline \multicolumn{4}{|l|}{ Anthropometric variables } \\
\hline Body mass index $\left(\mathrm{kg} / \mathrm{m}^{2}\right)$ & $28 \pm 6$ & $27 \pm 3$ & 0.5 \\
\hline Body surface area $\left(\mathrm{m}^{2}\right)$ & $1.9 \pm 0.2$ & $1.9 \pm 0.2$ & 0.9 \\
\hline \multicolumn{4}{|l|}{ Medical history } \\
\hline Time since commenced HD (days) & $94 \pm 84$ & & \\
\hline Diabetes mellitus (\%) & $14(26)$ & $2(7)$ & \\
\hline Ischaemic heart disease $(\%)$ & $13(24)$ & $0(0)$ & \\
\hline Current/ex-smoker (\%) & $25(46)$ & $8(28)$ & \\
\hline Peripheral vascular disease/stroke (\%) & $7(13)$ & $0(0)$ & \\
\hline LV systolic dysfunction (\%) & $4(7)$ & $0(0)$ & \\
\hline \multicolumn{4}{|l|}{ Medication } \\
\hline Treated hypertension $(\%)$ & $42(78)$ & $2(7)$ & \\
\hline RAAS antagonist (\%) & $14(26)$ & $1(3)$ & \\
\hline Beta blocker (\%) & $19(35)$ & $0(0)$ & \\
\hline Other antihypertensive (\%) & $37(69)$ & $2(7)$ & \\
\hline Statin use $(\%)$ & $23(43)$ & $4(14)$ & \\
\hline \multicolumn{4}{|l|}{ CMR parameters } \\
\hline Global peak systolic strain (\%) & $15.9 \pm 3.7$ & $19.3 \pm 3.4$ & $<0.001$ \\
\hline Basal peak systolic strain (\%) & $14.5 \pm 3.8$ & $18.4 \pm 4.5$ & $<0.001$ \\
\hline Mid-ventricular peak systolic strain(\%) & $16.2 \pm 4$ & $20 \pm 4.2$ & $<0.001$ \\
\hline Apical peak systolic strain (\%) & $16.9 \pm 4.1$ & $19.7 \pm 3.4$ & 0.002 \\
\hline Peak diastolic strain rate $\left(\mathrm{s}^{-1}\right)$ & $1.07 \pm 0.33$ & $1.31 \pm 0.38$ & 0.003 \\
\hline Peak systolic strain rate $\left(\mathrm{s}^{-1}\right)$ & $1.03 \pm 0.22$ & $1.28 \pm 0.24$ & 0.006 \\
\hline LV dyssynchrony by SD-TTP (ms) & $56 \pm 21$ & $39 \pm 14$ & $<0.001$ \\
\hline LV end-diastolic volume index $\left(\mathrm{ml} / \mathrm{m}^{2}\right)^{*}$ & $88 \pm 23$ & $75 \pm 14$ & 0.002 \\
\hline LV stroke volume index $\left(\mathrm{ml} / \mathrm{m}^{2}\right)^{*}$ & $44 \pm 12$ & $44 \pm 9$ & 0.8 \\
\hline LV ejection fraction $(\%)$ & $51 \pm 10$ & $59 \pm 5$ & $<0.001$ \\
\hline LV mass index $\left(\mathrm{g} / \mathrm{m}^{2}\right)^{*}$ & $63[54,79]$ & $46[42,53]$ & $<0.001$ \\
\hline LV mass/volume ratio $(\mathrm{g} / \mathrm{ml})$ & $0.76[0.64,0.86]$ & $0.65[0.55,0.71]$ & 0.001 \\
\hline Aortic distensibility $\left(\mathrm{mmHg}^{-1} \times 10^{-3}\right)$ & $2[1.3,3.1]$ & $4.1[3.1,6.2]$ & $<0.001$ \\
\hline Aortic pulse wave velocity $(\mathrm{m} / \mathrm{s})$ & $7.9 \pm 3.5$ & $5.3 \pm 1.9$ & 0.03 \\
\hline Systolic blood pressure (mmHg) & $143 \pm 27$ & $139 \pm 18$ & 0.6 \\
\hline Diastolic blood pressure $(\mathrm{mmHg})$ & $76 \pm 12$ & $80 \pm 10$ & 0.2 \\
\hline Pulse pressure (mmHg) & $61[50,79]$ & $60[48,68]$ & 0.3 \\
\hline
\end{tabular}

Categorical data are displayed as counts (percentages). Continuous data were tested for normality and summarized as mean \pm SD or median [25th, 75 th percentile]. HD, haemodialysis; LV, left ventricular; RAAS, reninangiotensin aldosterone system; SD-TTP standard deviation of time-to-peak strain.*Values indexed to body surface area. particularly in the septal and lateral walls (Table 3 and Fig. 4)

\section{LV dyssynchrony}

There was greater LV dyssynchrony in HD patients as determined by increased dispersion in the time to peak strain (SD-TTP $56 \pm 21 \mathrm{~ms}$ vs $39 \pm 14 \mathrm{~ms}, p<0.001$,
Table 2). LV dyssynchrony was inversely correlated to diastolic function by PDSR $(\mathrm{r}=-0.51, p<0.001)$ and $\mathrm{EF}$ $(-0.45, p=0.001$; Table 4).

\section{Aortic distensibility}

Aortic distensibility was decreased in HD patients $(2.0$ $[1.3,3.1]$ vs. $4.1[3.1,6.2], p<0.001)$ and correlated to 


\begin{tabular}{|c|c|c|c|c|c|}
\hline \multirow{17}{*}{$\begin{array}{l}\text { Table } 3 \text { Comparisons of } \\
\text { regional peak systolic } \\
\text { strain }(\%) \text { using the } 16 \\
\text { segment model of the left } \\
\text { ventricle. Values are } \\
\text { mean } \pm \text { SD }\end{array}$} & $\begin{array}{l}\text { LV segment } \\
\text { number }\end{array}$ & $\begin{array}{l}\text { LV segment } \\
\text { name }\end{array}$ & HD & $\mathrm{NC}$ & $p$ value \\
\hline & 1 & Basal Anterior & $13.7 \pm 5.7$ & $15.1 \pm 5.8$ & 0.3 \\
\hline & 2 & Basal Antero-septal & $9.6 \pm 5.2$ & $13.5 \pm 4.8$ & 0.001 \\
\hline & 3 & Basal Infero-septal & $11.3 \pm 5.1$ & $12.2 \pm 4.7$ & 0.4 \\
\hline & 4 & Basal Inferior & $14.7 \pm 6.8$ & $16.8 \pm 5.4$ & 0.2 \\
\hline & 5 & Basal Infero-lateral & $20.6 \pm 6.8$ & $24.5 \pm 5.8$ & 0.01 \\
\hline & 6 & Basal Antero-Lateral & $18.5 \pm 5.6$ & $22.5 \pm 5.6$ & 0.003 \\
\hline & 7 & Mid Anterior & $18.8 \pm 5.6$ & $21.6 \pm 4.3$ & 0.02 \\
\hline & 8 & Mid Antero-septal & $14.3 \pm 5.5$ & $18.0 \pm 4.5$ & 0.003 \\
\hline & 9 & Mid Infero-septal & $13.3 \pm 5.2$ & $18.0 \pm 3.5$ & $<0.001$ \\
\hline & 10 & Mid Inferior & $17.4 \pm 6.4$ & $20.0 \pm 4.9$ & 0.05 \\
\hline & 11 & Mid Infero-lateral & $23.3 \pm 6.4$ & $24.4 \pm 3.5$ & 0.4 \\
\hline & 12 & Mid Antero-Lateral & $19.3 \pm 4.9$ & $21.4 \pm 3.8$ & 0.05 \\
\hline & 13 & Apical Anterior & $17.5 \pm 4.9$ & $18.6 \pm 4.2$ & 0.3 \\
\hline & 14 & Apical Septal & $14.7 \pm 5.3$ & $18.0 \pm 4.1$ & 0.003 \\
\hline & 15 & Apical Inferior & $17.9 \pm 6.4$ & $20.4 \pm 6.7$ & 0.07 \\
\hline & 16 & Apical Lateral & $20.2 \pm 5.4$ & $20.7 \pm 4.8$ & 0.6 \\
\hline
\end{tabular}

PSS $(\mathrm{r}=0.37, p=0.047)$, PSSR $(\mathrm{r}=0.43, \mathrm{p}=0.02)$, highsensitivity troponin- $\mathrm{T}(\mathrm{r}=-0.53, \mathrm{p}=0.004)$ and concentric remodelling $(\mathrm{r}=-0.42, \mathrm{p}=0.02$, Table 4$)$. Aortic pulse wave velocity was increased in HD patients $(7.9 \pm 3.5$ versus $5.3 \pm 1.9 \mathrm{~m} / \mathrm{s}, p<0.001)$.

\section{Subgroup of HD patients with normal EF}

In the subset of HD patients with preserved $\mathrm{EF}>50 \%(\mathrm{n}=35)$, aortic distensibility was still reduced $\left(2.6[1.9,4.8] \mathrm{mmHg}^{-1} \times\right.$ $10^{-3}$ versus $\left.4.1[3.1,6.2] \mathrm{mmHg}^{-1} \times 10^{-3}\right)$, LV mass remained increased $\left(63[55,79] \mathrm{g} / \mathrm{m}^{2}\right.$ versus $46[42,53] \mathrm{g} / \mathrm{m}^{2}, p<$ $0.001)$, PSS was reduced $(17.5 \pm 3.1 \%$ versus $19.3 \pm 3.4 \%$, $p=0.03)$, PDSR was reduced $\left(1.10[0.83,1.31] \mathrm{s}^{-1}\right.$ versus $\left.1.27[1.04,1.41] \mathrm{s}^{-1}, p=0.03\right)$, and dyssynchrony remained greater (SD-TTP $51 \pm 18 \mathrm{~ms}$ versus $39 \pm 14 \mathrm{~ms}, p=0.006$; Table 5 and Fig. 5).

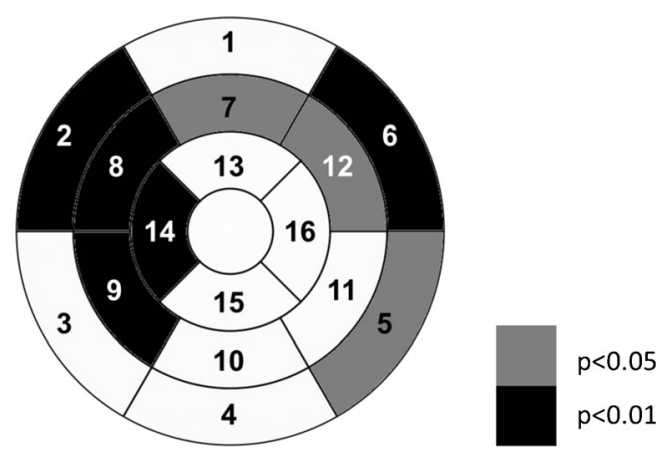

Fig. 4 Comparisons of regional PSS using the 16-segment model of the left ventricle. Segments with significantly lower PSS in HD patients compared to age-matched controls are graphically highlighted

\section{Subgroup of HD patients with diabetes, ischemic heart disease or $\mathrm{LV}$ dysfunction}

To account for the confounding of pre-existing cardiovascular co-morbidities, we conducted subgroup analyses amongst HD patients categorised by presence or absence of diabetes, ischemic heart disease, prior LV dysfunction, and use of a betablocker or renin-angiotensin aldosterone system antagonist. These results are detailed in Supplemental Table 1. As adverse changes were notable amongst HD patients with diabetes, we compared CMR parameters in non-diabetic HD patients $(\mathrm{n}=$ $40)$ to non-diabetic controls $(n=27)$. Abnormalities such as reduced systolic strain, increased LV mass and reduced aortic distensibility remained apparent in HD patients without diabetes (Supplemental Table 2).

\section{Discussion}

We report the first study using tagged CMR in patients within 6 months of commencing HD, characterising abnormalities in cardiac size, geometry and strain in terms of magnitude, rate and synchronicity. The high prevalence of reduced systolic and diastolic strain (despite preserved EF), ventricular dyssynchrony, anteroseptal segmental dysfunction and reduced aortic distensibility was more evident with the detailed methods utilised. The relative lack of correlation between bioimpedance-measured volume status and LV strain was consistent with volume-independent mechanisms playing a role in the evolution of cardiac dysfunction in this setting. 
Table 4 Correlation coefficients of systolic strain, diastolic strain-rate, LV dyssynchrony and aortic distensibility to potential determinants in HD patients

\begin{tabular}{|c|c|c|c|c|c|c|c|c|}
\hline \multirow[t]{2}{*}{ CMR Parameter } & \multicolumn{2}{|l|}{ PSS } & \multicolumn{2}{|l|}{ PDSR } & \multicolumn{2}{|c|}{ SD-TTP } & \multicolumn{2}{|c|}{ Aortic distensibility } \\
\hline & r-value & $p$ value & r-value & $p$ value & r-value & $p$ value & r-value & $p$ value \\
\hline Peak diastolic strain rate & 0.56 & $<0.001$ & - & - & -0.51 & $<0.001$ & 0.3 & 0.1 \\
\hline LV dyssynchrony by SD-TTP & -0.51 & $<0.001$ & -0.51 & $<0.001$ & - & - & -0.25 & 0.2 \\
\hline LV ejection fraction & 0.54 & $<0.001$ & 0.34 & 0.01 & -0.45 & 0.001 & 0.22 & 0.3 \\
\hline Global peak systolic strain rate & 0.53 & $<0.001$ & 0.6 & $<0.001$ & -0.35 & 0.01 & 0.43 & 0.02 \\
\hline LV mass index & -0.19 & 0.2 & -0.1 & 0.5 & 0.18 & 0.2 & -0.24 & 0.2 \\
\hline LV end-diastolic volume Index & 0.02 & 0.9 & 0.14 & 0.3 & 0.10 & 0.5 & 0.10 & 0.6 \\
\hline LV mass/volume ratio $(\mathrm{g} / \mathrm{ml})$ & -0.37 & 0.005 & -0.30 & 0.03 & 0.19 & 0.2 & -0.42 & 0.02 \\
\hline Global peak systolic strain & - & - & 0.56 & $<0.001$ & -0.51 & $<0.001$ & 0.37 & 0.05 \\
\hline Aortic distensibility ${ }^{\S}$ & 0.37 & 0.047 & 0.3 & 0.1 & -0.25 & 0.2 & - & - \\
\hline Aortic pulse wave velocity & 0.01 & 0.9 & -0.48 & 0.04 & 0.27 & 0.3 & -0.69 & 0.001 \\
\hline \multicolumn{9}{|l|}{ Biochemical markers } \\
\hline High-sensitivity troponin- $\mathrm{T}^{\S}$ & -0.41 & 0.03 & -0.18 & 0.4 & 0.37 & 0.06 & -0.53 & 0.004 \\
\hline NT-Pro-BNP ${ }^{\S}$ & -0.20 & 0.2 & -0.26 & 0.08 & 0.04 & 0.8 & -0.15 & 0.4 \\
\hline \multicolumn{9}{|l|}{ Demographic variables } \\
\hline Age & -0.24 & 0.08 & -0.31 & 0.02 & 0.07 & 0.6 & -0.73 & $<0.001$ \\
\hline \multicolumn{9}{|l|}{ Anthropometric variables } \\
\hline Body mass index & -0.12 & 0.4 & 0.05 & 0.7 & -0.08 & 0.6 & -0.09 & 0.6 \\
\hline Body surface area & -0.24 & 0.08 & 0.03 & 0.8 & 0.24 & 0.09 & -0.06 & 0.8 \\
\hline $\mathrm{ECW} / \mathrm{TBW}^{\S}$ & -0.26 & 0.1 & -0.18 & 0.3 & -0.03 & 0.8 & -0.28 & 0.2 \\
\hline
\end{tabular}

${ }^{\S}$ Log-transformed data

These data support and extend previous CMR-based descriptions of cardiomyopathy in established HD patients. Separate reports characterised different aspects of cardiac and aortic function; however, such a comprehensive assessment in incident HD patients has rarely been reported. Mark and coworkers reported gadolinium contrast-enhanced CMR data in 134 prevalent HD or peritoneal dialysis patients selected for cardiovascular screening for potential renal transplantation. They reported a prevalence of $72 \%$ for LV hypertrophy and $8.2 \%$ for reduced EF, using cut-offs derived from published reference range studies. They also found heterogeneity in the distribution of focal myocardial fibrosis as determined by gadolinium-based contrast enhancement. The study was conducted prior to awareness of the association of gadolinium contrast media with nephrogenic systemic fibrosis and did not investigate strain or relationships to troponin-T. Their findings were concordant with our study, demonstrating lower EF and greater LV mass in HD patients with regional heterogeneity in strain. The differences between these prior studies and the present might be explained by differences in the populations studied and exposure to HD.

There are no prior studies of LV strain by CMR tagging in HD patients, which is currently the reference standard technique for LV deformation. This present study reports strain values for the age and sex-matched controls without kidney disease $(19.5 \pm 3.3 \%)$ in keeping with reported reference ranges for circumferential strain by tagged CMR in healthy volunteers of $16-19 \%$ [23]. Strain values by tagged CMR may not be directly comparable to studies using tissue Doppler or speckle-tracking echocardiography. In general population studies, absolute differences of 10-25\% against a tagged CMR reference measure are reported, with wider disparities in disease states.

\section{Ventricular morphology}

Hypertrophy of the LV is an established feature of cardiomyopathy in HD patients, representing a maladaptive response that allows for normal EF despite abnormal pressure or volume load. In addition to traditional atherosclerotic risk factors, multiple factors are contributory, including aortic stiffness, volume and pressure overload, hyperphosphatemia and neurohormonal activation [24]. Recent work has extended this picture with the implication of locally generated steroids and fibroblast growth factor 23, both of which are increased in HD patients $[25,26]$. In particular, sodium can induce adrenal secretion of digitalis-like steroids to produce myocyte hypertrophy independent of blood pressure or volume effects, representing a novel mechanism linking dietary or dialysate sodium to LV hypertrophy in HD patients [27]. In the present 
Table 5 Subject characteristics and CMR parameters stratified by EF

\begin{tabular}{|c|c|c|c|}
\hline & $\begin{array}{l}\text { Controls with } \\
\mathrm{EF}>50 \%(\mathrm{n}=29)\end{array}$ & $\begin{array}{l}\text { HD with } \\
\mathrm{EF}>50 \%(\mathrm{n}=35)\end{array}$ & $p$ value \\
\hline Age (years) & $55 \pm 16$ & $56 \pm 17$ & 0.9 \\
\hline Female $(\%)$ & $9(26 \%)$ & $8(28 \%)$ & 1 \\
\hline \multicolumn{4}{|l|}{ Anthropometric variables } \\
\hline Body Mass Index $\left(\mathrm{kg} / \mathrm{m}^{2}\right)$ & $28 \pm 6$ & $27 \pm 3$ & 0.4 \\
\hline Body surface area $\left(\mathrm{m}^{2}\right)$ & $1.9 \pm 0.2$ & $1.9 \pm 0.2$ & 0.9 \\
\hline Extracellular water/Total body water ratio & $0.39[0.38,0.40]$ & & \\
\hline \multicolumn{4}{|l|}{ Medical history } \\
\hline Time since commenced HD (days) & $98 \pm 53$ & & \\
\hline Diabetes mellitus (\%) & $6(17)$ & $2(7)$ & \\
\hline Ischaemic heart disease $(\%)$ & $9(26)$ & $0(0)$ & \\
\hline Current/Ex-smoker (\%) & $15(43)$ & $8(28)$ & \\
\hline Peripheral vascular disease/Stroke (\%) & $4(11)$ & $0(0)$ & \\
\hline LV systolic dysfunction (\%) & $3(9)$ & $0(0)$ & \\
\hline \multicolumn{4}{|l|}{ Medication } \\
\hline Treated hypertension $(\%)$ & $29(83)$ & $2(7)$ & \\
\hline RAAS antagonist (\%) & $12(34)$ & $1(3)$ & \\
\hline Beta blocker $(\%)$ & $15(43)$ & $0(0)$ & \\
\hline Other antihypertensive $(\%)$ & $25(71)$ & $2(7)$ & \\
\hline Statin use $(\%)$ & $15(43)$ & $4(14)$ & \\
\hline \multicolumn{4}{|l|}{ Biochemical markers } \\
\hline High-sensitivity troponin- $\mathrm{T}(\mu \mathrm{g} / \mathrm{L})$ & $27[13,54]$ & & \\
\hline NT-pro-BNP (pg/ml) & $143[60,513]$ & & \\
\hline \multicolumn{4}{|l|}{ CMR parameters } \\
\hline Global peak systolic strain (\%) & $17.5 \pm 3.1$ & $19.3 \pm 3.4$ & 0.03 \\
\hline Basal peak systolic strain (\%) & $16.2 \pm 3.4$ & $18.4 \pm 4.5$ & 0.04 \\
\hline Mid-ventricular peak systolic strain (\%) & $18.0 \pm 3.4$ & $20.0 \pm 4.2$ & 0.05 \\
\hline Apical peak systolic strain (\%) & $18.2 \pm 3.9$ & $19.7 \pm 3.4$ & 0.1 \\
\hline Peak diastolic strain rate $\left(\mathrm{s}^{-1}\right)$ & $1.10[0.83,1.31]$ & $1.27[1.04,1.41]$ & 0.03 \\
\hline Peak systolic strain rate $\left(\mathrm{s}^{-1}\right)$ & $-1.05[-1.23,-0.89]$ & $-1.27[-1.40,-1.12]$ & $<0.001$ \\
\hline LV dyssynchrony by SD-TTP (ms) & $51 \pm 18$ & $39 \pm 14$ & 0.006 \\
\hline $\mathrm{LV}$ end-diastolic volume index $\left(\mathrm{ml} / \mathrm{m}^{2}\right)^{*}$ & $85 \pm 21$ & $75(14)$ & 0.04 \\
\hline LV stroke volume index $\left(\mathrm{ml} / \mathrm{m}^{2}\right)^{*}$ & $48 \pm 11$ & $44(9)$ & 0.2 \\
\hline LV ejection fraction $(\%)$ & $56 \pm 5$ & $59(5)$ & 0.01 \\
\hline LV mass index $\left(\mathrm{g} / \mathrm{m}^{2}\right)^{*}$ & $63[55,79]$ & $46[42,53]$ & $<0.001$ \\
\hline LV mass/volume ratio $(\mathrm{g} / \mathrm{ml})$ & $0.77[0.68,0.86]$ & $0.65[0.55,0.71]$ & $<0.001$ \\
\hline Aortic distensibility $\left(\mathrm{mmHg}^{-1} \times 10^{-3}\right)$ & $2.6[1.9,4.8]$ & $4.1[3.1,6.2]$ & 0.04 \\
\hline Aortic pulse wave velocity (m/s) & $7.0 \pm 3.6$ & $5.3 \pm 1.9$ & 0.2 \\
\hline Systolic blood pressure (mmHg) & $137 \pm 23$ & $139 \pm 18$ & 0.7 \\
\hline Diastolic blood pressure (mmHg) & $74 \pm 14$ & $80 \pm 10$ & 0.08 \\
\hline Pulse pressure $(\mathrm{mmHg})$ & $57[49,76]$ & $60[48,68]$ & 0.8 \\
\hline
\end{tabular}

Categorical data are displayed as counts (percentages). Continuous data were tested for normality and summarized as mean $\pm \mathrm{SD}$ or median $[25$ th, 75 th percentile]. HD, hemodialysis; LV, left ventricular; RAAS, renin-angiotensin aldosterone system; SD-TTP, standard deviation of time-to-peak strain.*Values indexed to body surface area.

ECW/TBW extracellular water/total body water by bioimpedance

NT-pro-BNP N-Terminal Pro-B-type natriuretic peptide

RAAS Renin-angiotensin aldosterone system

SD-TTP standard deviation of the segmental time to peak circumferential strain 


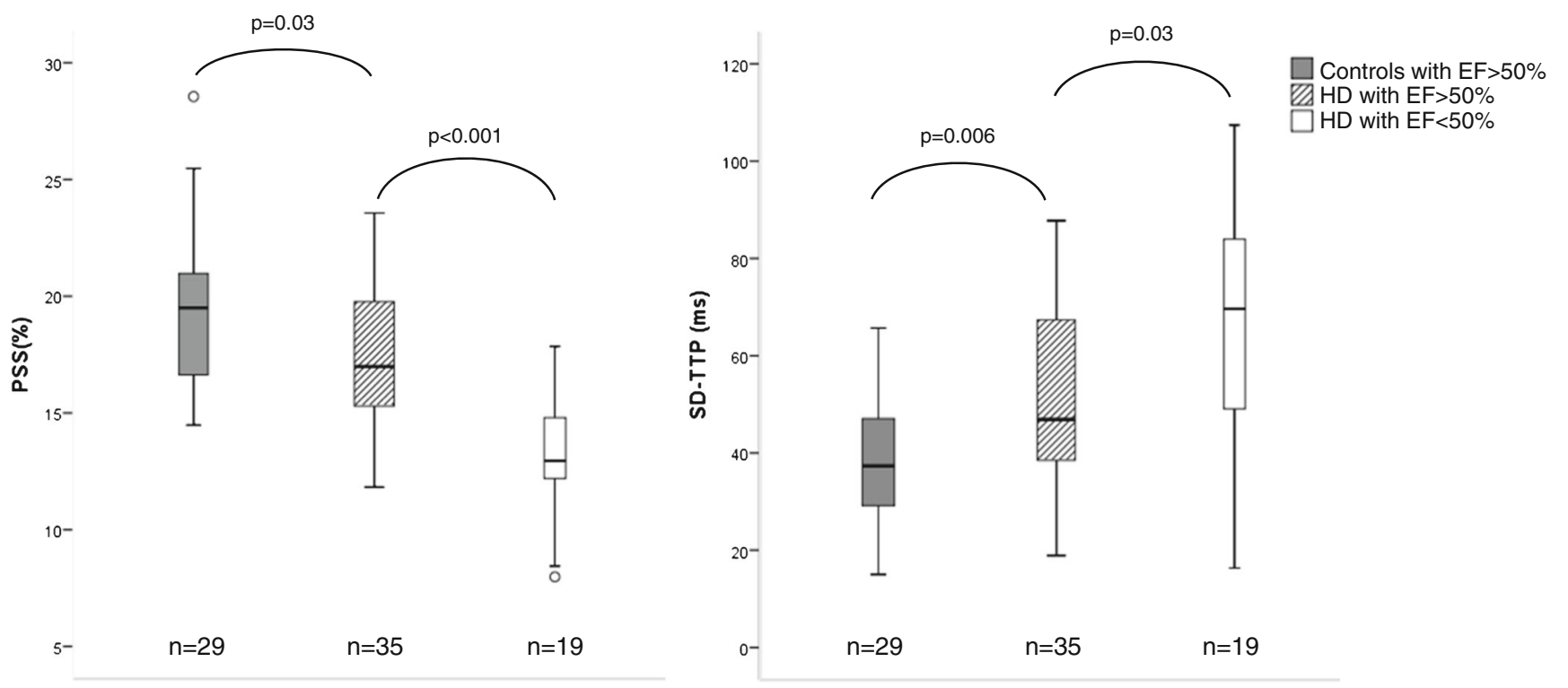

Fig. 5 Boxplots of Global PSS and LV dyssynchrony by SD-TTP stratified by EF

study, LV concentric remodelling by mass/volume ratio was significantly increased in HD patients compared to controls, even amongst those with preserved EF or without diabetes.

\section{Heterogeneity of systolic strain}

The regional heterogeneity of PSS has not previously been described in HD patients. In studies of hypertensive cardiomyopathy, tagged CMR demonstrated the earliest effects of pressure overload by basal septal reductions in PSS and PSSR [28]. This is in contrast to infiltrative genetic disorders such as Fabry's disease where the earliest reductions in PSS are seen in inferolateral segments [29]. The present study reports reductions in the entire septum, as well as anterolateral segments. This supports the notion that HD might be associated with a distinct cardiomyopathy, reflecting several remodelling signals, some of which are independent of pressure or volume, including recurrent $\mathrm{HD}$ ischemic insults [24,30]. We observed significant correlations of PSS to LV dyssynchrony, concentric remodelling, aortic distensibility, aortic stiffness and high sensitivity troponin- $\mathrm{T}$.

\section{Diastolic function}

The determinants of diastolic function in HD patients are not well described, despite an increasing appreciation of the prognostic importance of this form of ventricular relaxation defect. Using speckle tracking echocardiography, De Bie and coworkers reported univariate determinants as LV mass, concentric remodelling and PWV [31]. Losi and co-workers found a significant relationship of diastolic dysfunction in HD patients to echocardiographic-integrated backscatter (as a surrogate for myocardial fibrosis) [32]. We additionally report significant relationships to LV dyssynchrony and age.

\section{Synchronicity and coherence of ventricular contraction}

Synchronous contraction of the LV is essential to efficient cardiac output. LV dyssynchrony by tagged CMR has not previously been described in HD patients; in other settings, good reproducibility, preload independence and the ability to predict benefit from resynchronisation therapies have been demonstrated [18, 33]. El Ghannudi and co-workers reported SD-TTP of $28.5 \pm 11.5 \mathrm{~ms}$ in control subjects and $91.3 \pm 25 \mathrm{~ms}$ in patients with $\mathrm{EF}<55 \%$ due to ischemic or dilated cardiomyopathy. This compares with $39 \pm 14 \mathrm{~ms}$ in control subjects and $56 \pm 21 \mathrm{~ms}$ in HD patients in the present study. LV dyssynchrony was positively correlated to PSS and inversely correlated to diastolic function by PDSR $(\mathrm{r}=-0.51, p<0.001)$ and EF $(-0.45, \mathrm{p}=0.001)$. This study is the first to link LV dyssynchrony and diastolic dysfunction in HD patients.

\section{Aortic distensibility}

Aortic distensibility was reduced in HD patients and this pattern persisted in the subgroup of HD patients with preserved EF or without diabetes. Reported values are consistent with prior studies [34]. In the present study, the strong correlation of aortic distensibility to troponin- $\mathrm{T}(\mathrm{r}=-0.53, p=0.004)$ is novel. Troponin-T reflects the presence and severity of HDinduced ischemia [35], and is potentially aggravated by the increased risk of demand ischemia resulting from reduced aortic distensibility causing reduced diastolic coronary blood flow [36]. Given that we examined an incident population which had been exposed to HD for a mean of 98 days, it is 
likely that the changes we saw in HD patients occurred, at least in part, prior to commencing HD. Edwards et al. compared aortic distensibility by CMR in patients with CKD stage 3 to normal controls [37]. They derived similar values to the present study $\left(2.2 \pm 1.8\right.$ in CKD vs. $4.1 \pm 1.3 \mathrm{mmHg}^{-1} \times 10^{-3}$ in controls) compared with $2.0[1.3,3.1]$ vs. $4.1[3.1,6.2]$ in the present study).

\section{Volume status}

Biomarkers of volume status (ECW/TBW, LV end-diastolic volume index and NT-pro-BNP) showed no significant correlations to systolic strain, diastolic strain rate, LV dyssynchrony or aortic distensibility. This might indirectly represent volume-independent influences on cardiac function in HD patients.

\section{Limitations}

Although we have examined associations, the cross-sectional design limits our ability to determine any causal relationships. In particular, a longitudinal study design would be a better design to assess the impact of HD on the natural history of cardiomyopathy. Circumferential strain is reported, but the lack of longitudinal strain is a limitation. Furthermore, we did not have a CKD or treated hypertension control group and, thus, cannot determine the extent of their relative contributions to the observed between group differences. This could be addressed by a longitudinal study design from early-stage CKD through to HD, but as most patients with CKD do not progress to end-stage kidney disease [38], such a study would be prohibitively large. An alternative longitudinal studies amongst matched groups with hypertension, CKD and incident HD. Such a study might still have residual confounding that might not entirely be removed by statistical methods.

\section{Conclusion}

This study provides the first data comprehensively characterising LV strain, dyssynchrony and aortic distensibility by tagged CMR imaging in patients new to HD, revealing a greater degree of cardiac dysfunction than previously recognized. These abnormalities still persisted in HD patients with preserved EF or without diabetes. This reinforces the need to proactively engage in cardio-protective strategies and stresses the particular potential of dialysis therapy-based interventions.

Acknowledgements The scientific guarantor of this publication is Dr Odudu. Dr Horsfield declares a directorship of Xinapse Systems (imaging software). The remaining authors of this manuscript declare no relationships with any companies whose products or services may be related to the subject matter of the article. This study was funded in part by a National Institute for Health Research Grant (PB-PG-0408-16195) to
Dr. McIntyre. Dr Odudu acknowledges support of a British Heart Foundation Research Training Fellowship grant (Ref: FS/11/10/28564) and a National Institute for Health Research Clinical Lectureship. Dr Breidthardt was supported by a Swiss National Science Foundation Research grant. Dr. McCann is supported by a National Institute for Health Research post-doctoral research fellowship (PDF-2011-04-51). No complex statistical methods were necessary for this paper. The study protocol was reviewed by the Derby Hospitals' NHS Foundation Trust statistician before submission to the institutional review board. Institutional review board approval was obtained from the Nottingham Ethics Committee (09/ H0408/71). Some patients have been previously reported in The Clinical Journal of the American Society of Nephrology.

Written informed consent was obtained from all subjects (patients) in this study. Methodology: prospective, case-control study, multicenter study.

Open Access This article is distributed under the terms of the Creative Commons Attribution-NonCommercial 4.0 International License (http:// creativecommons.org/licenses/by-nc/4.0/), which permits any noncommercial use, distribution, and reproduction in any medium, provided you give appropriate credit to the original author(s) and the source, provide a link to the Creative Commons license, and indicate if changes were made.

\section{References}

1. Gross ML, Ritz E (2008) Hypertrophy and fibrosis in the cardiomyopathy of uremia - Beyond coronary heart disease. Semin Dial 21:308-318

2. Palmer SC, Craig JC, Navaneethan SD, Tonelli M, Pellegrini F, Strippoli GF (2012) Benefits and harms of statin therapy for persons with chronic kidney disease: a systematic review and metaanalysis. Ann Intern Med 157:263-275

3. Chertow GM, Levin NW, Beck GJ et al (2010) In-center hemodialysis six times per week versus three times per week. N Engl J Med 363:2287-2300

4. Selby NM, Burton JO, Chesterton LJ, McIntyre CW (2006) Dialysis-induced regional left ventricular dysfunction is ameliorated by cooling the dialysate. Clin J Am Soc Nephrol 1:1216-1225

5. Zoccali C, Benedetto FA, Mallamaci F et al (2004) Left ventricular mass monitoring in the follow-up of dialysis patients: prognostic value of left ventricular hypertrophy progression. Kidney Int 65 : $1492-1498$

6. Bluemke DA, Kronmal RA, Lima JAC et al (2008) The Relationship of Left Ventricular Mass and Geometry to Incident Cardiovascular Events. J Am Coll Cardiol 52:2148-2155

7. Shehata ML, Cheng S, Osman NF, Bluemke DA, Lima JA (2009) Myocardial tissue tagging with cardiovascular magnetic resonance. J Cardiovasc Magn Reson 11:55

8. Lukowsky LR, Kheifets L, Arah OA, Nissenson AR, KalantarZadeh K (2012) Patterns and predictors of early mortality in incident hemodialysis patients: new insights. Am J Nephrol 35:548558

9. Mark PB, Johnston N, Groenning BA et al (2006) Redefinition of uremic cardiomyopathy by contrast-enhanced cardiac magnetic resonance imaging. Kidney Int 69:1839-1845

10. Movilli E, Cancarini GC, Cassamali S et al (2004) Inter-dialytic variations in blood volume and total body water in uraemic patients treated by dialysis. Nephrol Dial Transplant 19:185-189

11. Maceira AM, Prasad SK, Khan M, Pennell DJ (2006) Normalized left ventricular systolic and diastolic function by steady state free 
precession cardiovascular magnetic resonance. J Cardiovasc Magn Reson 8:417-426

12. Fürstenberg A, Davenport A (2011) Comparison of Multifrequency Bioelectrical Impedance Analysis and Dual-Energy X-ray Absorptiometry Assessments in Outpatient Hemodialysis Patients. Am J Kidney Dis 57:123-129

13. Kyle UG, Bosaeus I, De Lorenzo AD et al (2004) Bioelectrical impedance analysis - part I: review of principles and methods. Clin Nutr 23:1226-1243

14. Giannitsis E, Kurz K, Hallermayer K, Jarausch J, Jaffe AS, Katus HA (2010) Analytical Validation of a High-Sensitivity Cardiac Troponin T Assay. Clin Chem 56:254-261

15. Papavassiliu T, Kuhl HP, Schroder M et al (2005) Effect of endocardial trabeculae on left ventricular measurements and measurement reproducibility at cardiovascular MR imaging. Radiology 236:57-64

16. Mosteller RD (1987) Simplified calculation of body-surface area. $\mathrm{N}$ Engl J Med 317:1098

17. Miller CA, Borg A, Clark D et al (2013) Comparison of local sine wave modeling with harmonic phase analysis for the assessment of myocardial strain. J Magn Reson Imaging 38:320-328

18. El Ghannudi S, Germain P, Jeung MY et al (2014) Quantification of left ventricular dyssynchrony in patients with systolic dysfunction: a comparison of circumferential strain MR-tagging metrics. J Magn Reson Imaging 40:1238-1246

19. Cerqueira MD, Weissman NJ, Dilsizian V et al (2002) Standardized Myocardial Segmentation and Nomenclature for Tomographic Imaging of the Heart: A Statement for Healthcare Professionals From the Cardiac Imaging Committee of the Council on Clinical Cardiology of the American Heart Association. Circulation 105: 539-542

20. Bramwell JC, Hill AV (1922) Velocity of Transmission of the Pulse-Wave. The Lancet 199:891-892

21. Fielden SW, Fornwalt BK, Jerosch-Herold M, Eisner RL, Stillman AE, Oshinski JN (2008) A new method for the determination of aortic pulse wave velocity using cross-correlation on 2D PCMR velocity data. J Magn Reson Imaging 27:1382-1387

22. Benjamini Y, Krieger AM, Yekutieli D (2006) Adaptive linear stepup procedures that control the false discovery rate. Biometrika 93: 491-507

23. Moore CC, Lugo-Olivieri CH, McVeigh ER, Zerhouni EA (2000) Three-dimensional systolic strain patterns in the normal human left ventricle: characterization with tagged MR imaging. Radiology 214:453-466

24. Glassock RJ, Pecoits-Filho R, Barberato SH (2009) Left Ventricular Mass in Chronic Kidney Disease and ESRD. Clin J Am Soc Nephrol 4:S79-S91
25. Stella P, Manunta P, Mallamaci F et al (2008) Endogenous ouabain and cardiomyopathy in dialysis patients. J Intern Med 263:274-280

26. Gutierrez OM, Januzzi JL, Isakova T et al (2009) Fibroblast growth factor 23 and left ventricular hypertrophy in chronic kidney disease. Circulation 119:2545-2552

27. Bagrov AY, Fedorova OV (2005) Cardenolide and bufadienolide ligands of the sodium pump. How they work together in $\mathrm{NaCl}$ sensitive hypertension. Front Biosci 10:2250-2256

28. Baltabaeva A, Marciniak M, Bijnens B et al (2008) Regional left ventricular deformation and geometry analysis provides insights in myocardial remodelling in mild to moderate hypertension. Eur $\mathrm{J}$ Echocardiogr 9:501-508

29. Bijnens BH, Cikes M, Claus P, Sutherland GR (2009) Velocity and deformation imaging for the assessment of myocardial dysfunction. Eur J Echocardiogr 10:216-226

30. Shivalkar B, Flameng W, Szilard M, Pislaru S, Borgers M, Vanhaecke J (1999) Repeated stunning precedes myocardial hibernation in progressive multiple coronary artery obstruction. J Am Coll Cardiol 34:2126-2136

31. de Bie MK, Ajmone Marsan N, Gaasbeek A et al (2012) Left ventricular diastolic dysfunction in dialysis patients assessed by novel speckle tracking strain rate analysis: prevalence and determinants. Int J Nephrol 2012:963504

32. Losi MA, Memoli B, Contaldi C et al (2010) Myocardial fibrosis and diastolic dysfunction in patients on chronic haemodialysis. Nephrol Dial Transplant 25:1950-1954

33. Helm RH, Lardo AC (2008) Cardiac magnetic resonance assessment of mechanical dyssynchrony. Curr Opin Cardiol 23:440-446

34. Zimmerli LU, Mark PB, Steedman T et al (2007) Vascular function in patients with end-stage renal disease and/or coronary artery disease: a cardiac magnetic resonance imaging study. Kidney Int 71:68-73

35. Breidthardt T, Burton JO, Odudu A, Eldehni MT, Jefferies HJ, McIntyre CW (2012) Troponin $T$ for the detection of dialysisinduced myocardial stunning in hemodialysis patients. Clin J Am Soc Nephrol 7:1285-1292

36. Ohtsuka S, Kakihana M, Watanabe H, Sugishita Y (1994) Chronically decreased aortic distensibility causes deterioration of coronary perfusion during increased left ventricular contraction. J Am Coll Cardiol 24:1406-1414

37. Edwards NC, Ferro CJ, Townend JN, Steeds RP (2008) Aortic distensibility and arterial-ventricular coupling in early chronic kidney disease: a pattern resembling heart failure with preserved ejection fraction. Heart 94:1038-1043

38. Sud M, Tangri N, Levin A, Pintilie M, Levey AS, Naimark DM (2014) CKD Stage at Nephrology Referral and Factors Influencing the Risks of ESRD and Death. Am J Kidney Dis 63:928-936 